\title{
Adynamic Donor Ureter: A Case Report with Review of the Literature
}

\author{
Jalbani IK ${ }^{1}$, Memon $\mathrm{A}^{1}$ and Khan TFT ${ }^{\star 2}$
}

${ }^{1}$ Department of Urology, Aga Khan University Hospital, Karachi, Paskistan

${ }^{2}$ Department of Surgery, North West Armed Forces Hospital, Tabuk, Kingdom of Saudi Arabia

*Corresponding author: Khan TFT, Department of Surgery, Section of Kidney Transplantation, Rehman Medical Institute, Hayatabad, Peshawar, Pakistan, Tel: 00923034455734, E-mail: taqikahn@yahoo.com

Citation: Jalbani IK, Memon A, Khan TFT (2018) Adynamic Donor Ureter: A Case Report with Review of the Literature. J Nephrol Kidney Dis 1(1): 102. doi: 10.15744/2767-9225.1.102

Received Date: September 10, 2018 Accepted Date: April 27, 2020 Published Date: April 29, 2020

\begin{abstract}
We report a unique case of a male recipient who received a zero mismatch kidney with mild anatomical anomalies from his sister. This right kidney was malrotated, had an extra renal pelvis with moderately dilated ureter up to the iliac artery bifurcation. Immediate graft function was achieved on reperfusion, and the recipient was discharged on day 7 with a serum creatinine (SC) of $1.8 \mathrm{mg} / \mathrm{dl}$. The DJ stent was removed after 6 weeks, with a rapid increase in SC to $3.5 \mathrm{mg} / \mathrm{dl}$, and ultrasound showed mild hydronephrosis with delayed excretion on nuclear imaging. Mechanical obstruction was excluded by retrograde pyelography, and a 5F DJ stent was placed across the ureterovesical anastomosis with an immediate decrease in SC. The case is presented in detail and discussed in the light of the current literature.
\end{abstract}

Keywords: Adynamic Ureter; Hydroureter; DJ Stenting; Kidney Transplantation

\section{Introduction}

Organ shortage is universal problem and more than 124, 000 patients are wait-listed for organs in the United States, and this number increases annually despite patients who die or become unfit for transplantation [1]. Efforts have been made to increase organ pool from deceased donation, donation after cardiac death, paired exchange, altruistic and marginal donors. This condition is worse in developing countries, where organ donation is almost non-existing [2]. Coping with this has resulted in the acceptance of living donor kidneys with anomalies besides greater acceptance of marginal and suboptimal graft from deceased donors [3,4]. This has compelled transplant centers to accept organs that would otherwise not have been accepted, and also includes organs with unusual anatomy such as horseshoe kidneys or a graft with a double ureter [4,5]. Herein we report a unique case of a male recipient who received a zero mismatch anomalous kidney from his sister. This right kidney was malrotated, had mild hydronephrosis, an extrarenal pelvis, and a moderate hydroureter up to the iliac artery bifurcation.

\section{Case Report}

A 43 years old male with end stage renal disease secondary to hypertension on regular hemodialysis for 2 years was referred for kidney transplantation. The past history was essentially unremarkable, apart from controlled hypertension. The family history was significant for hypertension, diabetes and ischemic heart disease. On presentation his BMI was 28.9, otherwise his general physical and systemic examinations were unremarkable. The donor was his 36 years old sister, with BMI of 22.71 and a GFR was $113 \mathrm{ml} /$ $\mathrm{min} / 1.73 \mathrm{~m}^{2}$, a zero HLA mismatch and IGg positive for cytomegalovirus (CMV).

Computerized Tomography scanning showed a malrotated right kidney, an extra renal pelvis with moderately dilated ureter up to the iliac artery bifurcation, and was suggestive of a radiolucent calculus (Figure 1). Isotope uptake by this kidney was $46 \%$ on nuclear scanning without any evidence of outflow obstruction (Figure 2). The presumed radiolucent calculus in the distal ureter was deemed inconsequential, because, at recovery, this ureter would be divided proximal to it. Based on the above results, this kidney was selected for implantation, and both recipient and donor received appropriate counselling.

Since this was a zero HLA mismatch case, only a single dose of $1.5 \mathrm{mg} / \mathrm{Kg}$ Thymoglobulin and a daily dose of $250 \mathrm{mg}$ of Methylprednisolone were given for 4 days with no plan for oral steroids. The kidney was implanted extraperitoneally on the right side with end to side vascular anastomosis to the external iliac vessels, and an extravesical neoureterocystostomy was fashioned over a double J stent (DJ). Immediate graft function was achieved, and the serum creatinine (SC) dropped rapidly to $1.8 \mathrm{mg} / \mathrm{dl}$ 
from $7.3 \mathrm{mg} / \mathrm{dl}$ in the first 48 hours. Post-operative Doppler ultrasound (US) and nuclear scan were within normal limits and the Foley catheter was removed on day 5 after surgery (Figure 3). The patient was discharged home on post-op day 7, on Tacrolimus and Cellcept without glucocorticoids, along with universal CMV prophylaxis, and the SC stabilized at around 1.5mg/dl. The DJ stent was removed as per protocol at 6 weeks post-transplant and the SC increased to $3.5 \mathrm{mg} / \mathrm{dl}$ in 3 days, and US showed moderate hydronephrosis and no outflow obstruction was found on nuclear imaging (Figure 4). Tacrolimus levels were acceptable, and all inflammatory markers were normal and hydration did not improve the renal function.
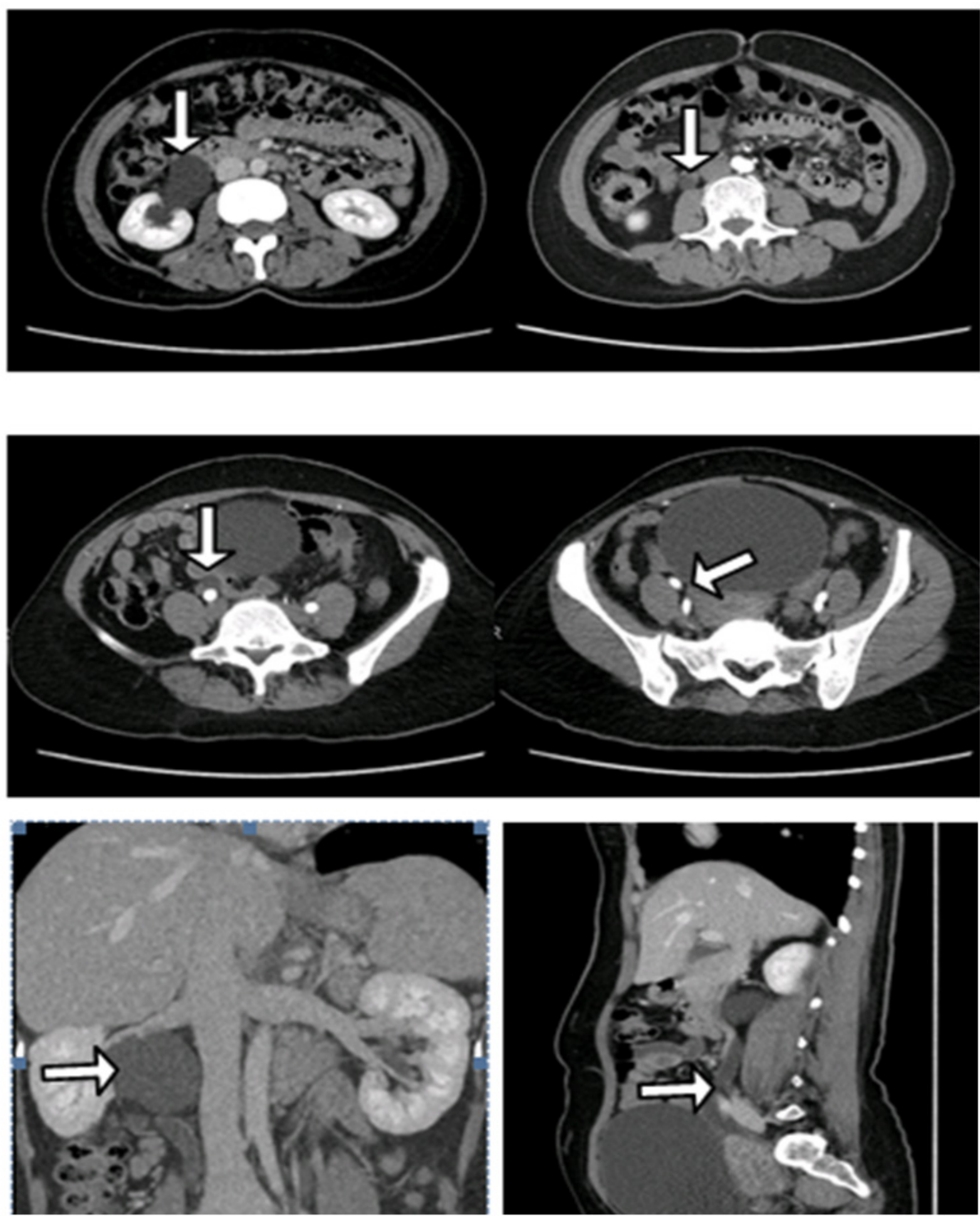

Figure 1: Donor CT scan abdomen with intravenous contrast: Axial sections: There is mild hydronephrosis extrarenal pelvis noted on right side with hydroureter(vertical arrows) noted till bifurcation of common iliac artery, thereafter, no hydroureter is noted (oblique arrow). Similar findings are noted on coronal \& sagital images (horizontal arrows) 


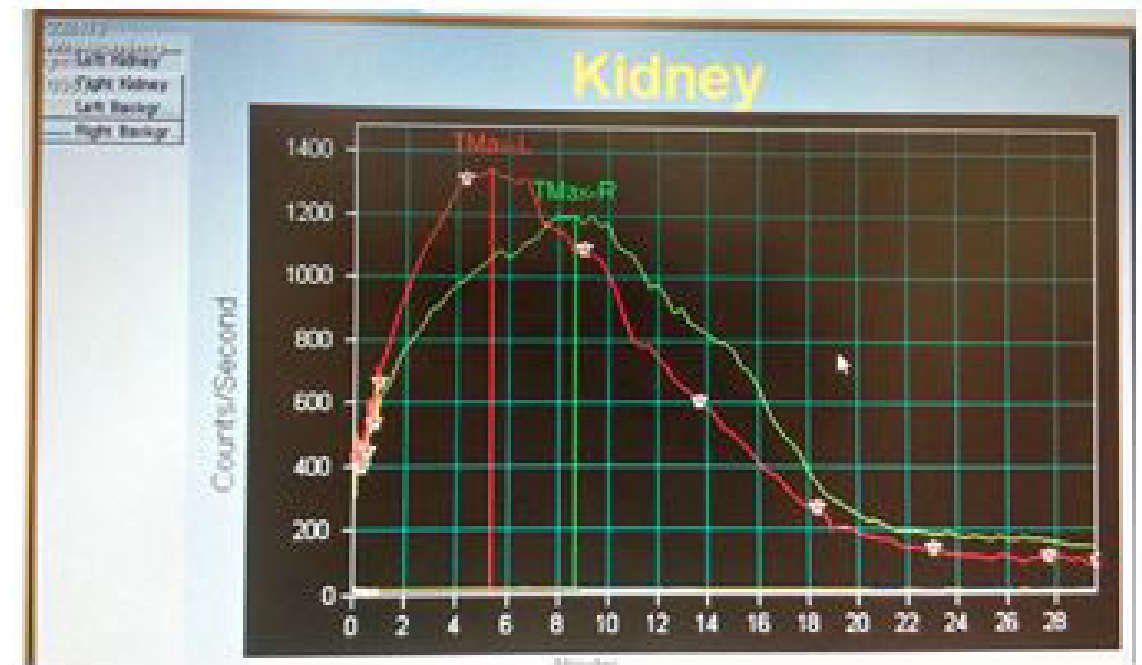

Figure 2: MAG III: Showing good uptake and clearence of istope without any evidence of outflow obstruction

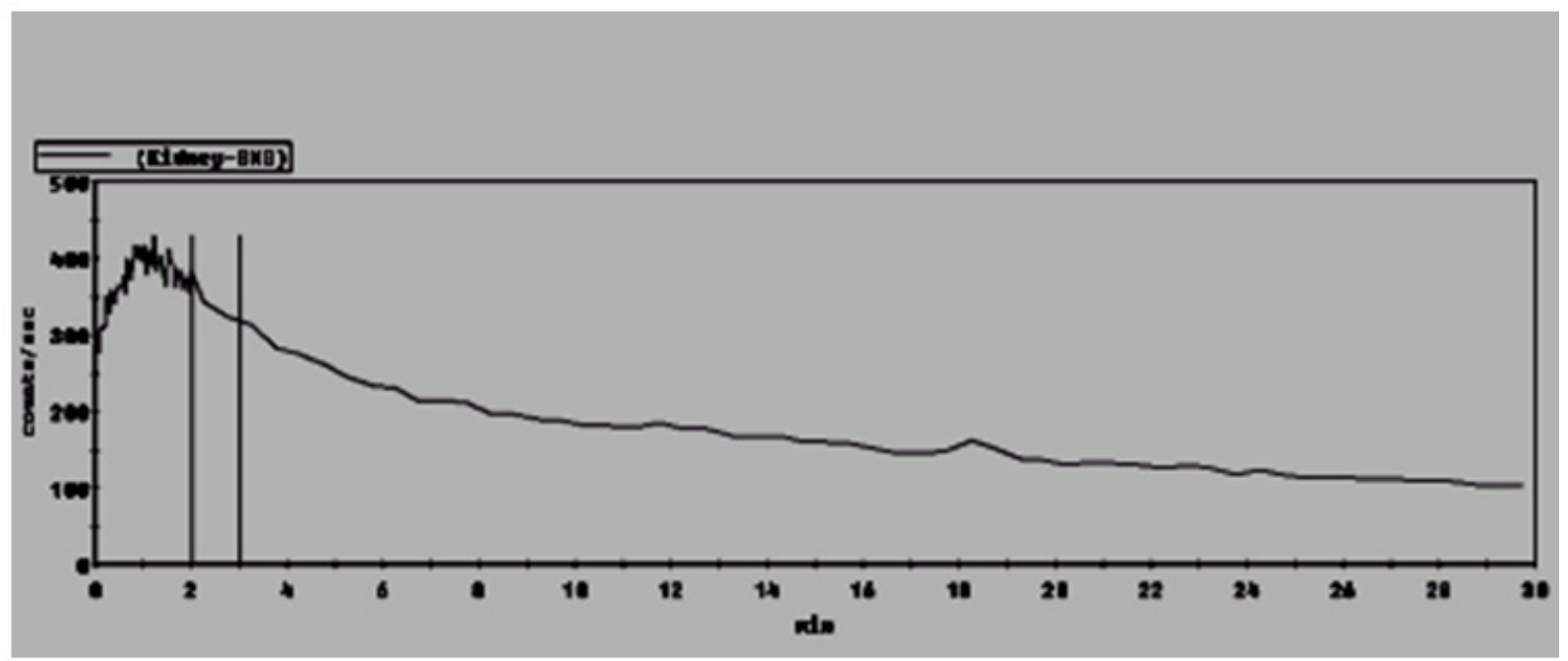

Figure 3: MAG III Day 2 post kidney transplant, Good uptake and clearence of isotope is noted

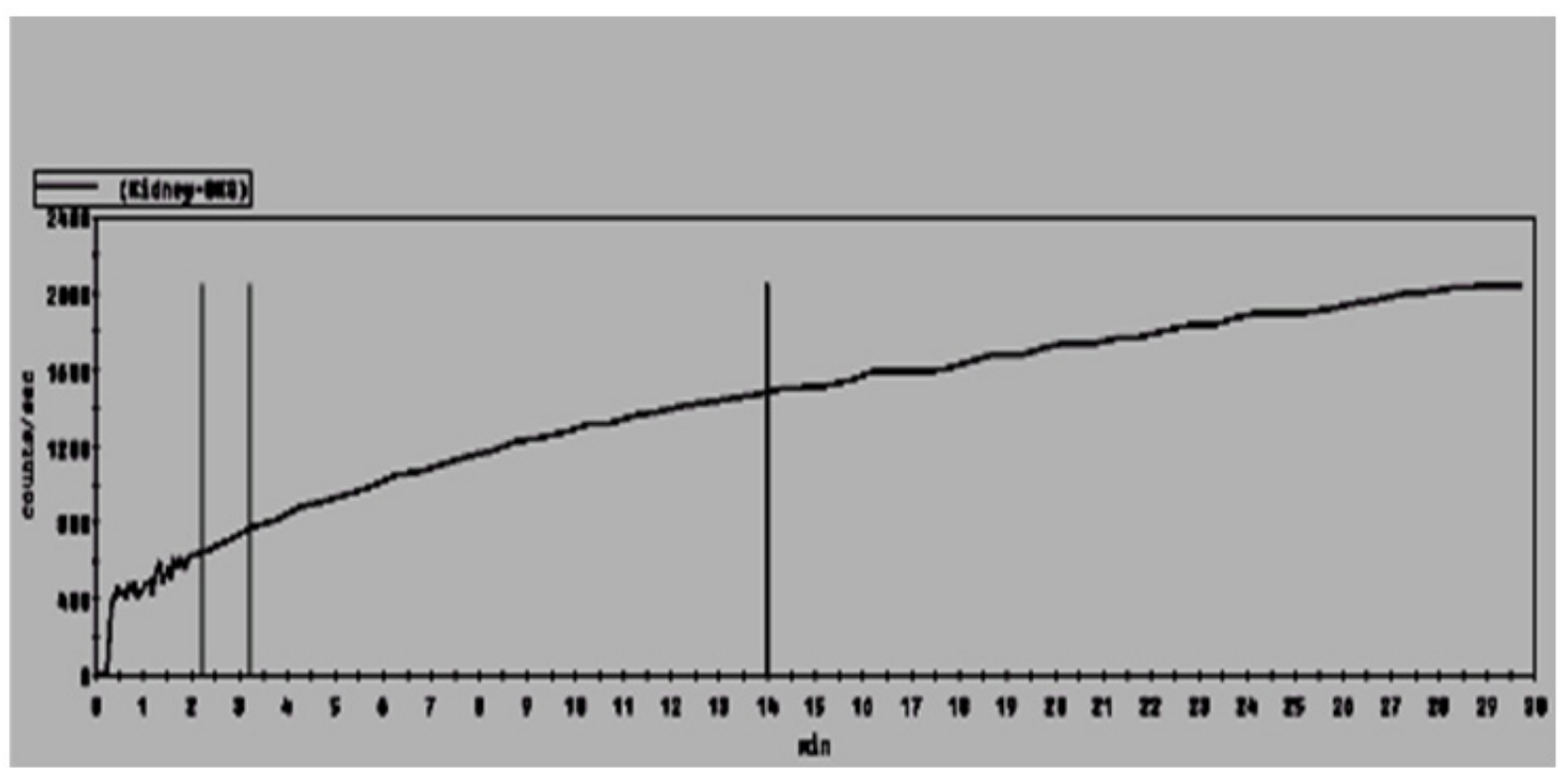

Figure 4: MAG III scan: Two days after removal of DJ stent removal, isotope uptake is adequate, but with very little clearance 
The rise in SC after removal of the DJ stent and the new onset hydronephrosis on US was considered mechanical obstruction, but this was excluded by retrograde pyelography and a 5F DJ was placed. The absence of stenosis or a calculus in the presence of the dilated ureter was suggestive of an adynamic donor ureter, and was indirectly confirmed by the immediate decrease in SC to $2.5 \mathrm{mg} / \mathrm{dl}$, and at 4 -month follow-up, the SC was stable at $1.8-2 \mathrm{mg} / \mathrm{dl}$ with a planned stent exchange every 6 months. The alternative to stent exchange of pyeloureterostomy that would have avoided repeated intervention was discussed with the patient, but he remained undecided. A year later however, despite aggressive antibiotic therapy, ventilator and inotropic support, the patient succumbed to a fulminant pneumonia with a functioning graft, without any evidence of urinary sepsis.

\section{Discussion}

Ureteral complications after renal transplantation have been attributed primarily to technical problems during donor surgery and implantation. Vesicoureteral junction is commonest site of stenosis post kidney transplant, and is managed initially with percutaneous nephrostomy to prevent any adverse impact on the graft, ureteral stenting and balloon dilatation (antegrade) are other available options [6,7]. To the best of our knowledge, this is the first report in the literature of an adynamic ureter in a donor kidney. The commonest reported anomaly is a horseshoe kidney, that generally requires division, and the most frequent complication is a urinary leak because of the anterior location of the renal pelvis [8].

This right kidney was considered acceptable despite its anomalies because it was the smaller of the two kidneys on split function (46\%), and also because it had the unexplained hydroureter. Adynamic donor ureter was a post-transplant diagnosis of exclusion because of the increase in SC after stent removal, and the lack of evidence of mechanical obstruction on retrograde pyelography. Further indirect confirmation of the diagnosis was provided by the rapid decrease in SC after placement of a DJ stent. At last follow up, the renal function was stable, and no back pressure changes were evident on US. Ideally, the diagnosis of adynamic ureter required histological confirmation, because a ureter specimen generally becomes available when the distal end of the donor ureter is freshened, but unfortunately our specimen was lost.

\section{Conclusion}

A diagnosis of an adynamic ureter in donor kidneys should be suspected in the presence of other anomalies such as malrotation and hydronephrosis without obstruction. Despite this, the affected kidney should be considered for transplantation because of the universal organ shortage. Transplant surgeons should be familiar with this condition, and donor and recipient require appropriate counseling for the possibility of long-term replacement of indwelling ureteric stents and antibiotic prophylaxis.

\section{References}

1. Organ Procurement and Transplantation Network, USA.

2. Rizvi SA, Naqvi SA, Hussain Z, Hashmi A, Akhtar F, et al. (2003) Renal transplantation in developing countries. Kidney Int 63: S96-S100.

3. Lempinen M, Stenman J, Kyllönen L, Salmela K (2015) Surgical complications following 1670 consecutive adult renal transplantations: A single center study. Scand J Surg 104: 254-9.

4. Justo-Janeiro JM, Orozco EP, Reyes FJ, de la Rosa Paredes R, de Lara Cisneros LG, et al. (2015) Transplantation of a horse shoe kidney from a living donor. Case report, long term outcome and donor safety. Int J Surg Case Rep 15: 21-5.

5. Rodriguez MM (2014) Congenital anomalies of the kidney and the urinary tract (CAKUT). Fetal Pediatr Pathol 33: 293-20.

6. Duty BD, Barry JM (2015) Diagnosis and management of ureteral complications following renal transplantation. Asian J Urol 2: $202-7$.

7. Halstuch D, Ehrlich Y, Shenhar C, Mano R, Baniel J, et al. (2018) Transplant Kidney Retrograde Ureteral Stent Placement and Exchange: Overcoming the Challenge. Urol 1: 220-4.

8. Goyal A, Gaitonde K, Sagade SN, Shah BV, Kamat MH (2003) Transplantation of horseshoe kidney from living-related donors: report of two cases. Transplant Proc 35: 32-4. 